\title{
Yellow nails, lymphoedema, and pleural effusions
}

\author{
PETER A. EMERSON \\ From Westminster Hospital, London, S.W.1
}

The purpose of this paper is to draw attention to a new syndrome and to describe three examples of chronic pleural effusion due to chronic lymphoedema. Before encountering these patients I had never previously considered chronic lymphoedema as a cause of chronic pleural effusion: it is certainly not described in any standard textbook, although it was tentatively referred to in one in 1963 (Robson and Emerson, 1963), and more recently Hurwitz and Pinnals (1964) have described two patients with pleural effusions and lymphoedema only.

The thesis is that an otherwise unexplained pleural effusion may occur in patients with chronic lymphoedema and be due to some deficiency in the lymphatics draining the pleural space. These two conditions may be associated with yellow finger- and toe-nails, due also to impaired lymphatic drainage.

\section{CASE REPORTS}

CASE 1 Miss M. J., born in 1923, was known from infancy to have had swelling of the legs and to a lesser extent of the backs of the hands with puffiness of the face. There was no family history of lymphoedema, and apart from the swelling she was well except for occasional attacks of bronchitis. These all cleared up completely until one attack in 1954, after which she became, and remained, short of breath, and was subsequently found to have a large right and a small left pleural effusion (Fig. 1). Over the subsequent years until her death in 1960 from an unrelated cause (malignant melanoma) the effusions persisted and recurred soon after each of the innumerable aspirations which were carried out.

The pleural fluid was always clear, straw-coloured material ; the predominant cell was the lymphocyte, with occasional histiocytes and mesothelial cells. The protein content was usually 4.5 to $5 \mathrm{~g} . / 100 \mathrm{ml}$., and malignant cells were never seen. The albumin turn-

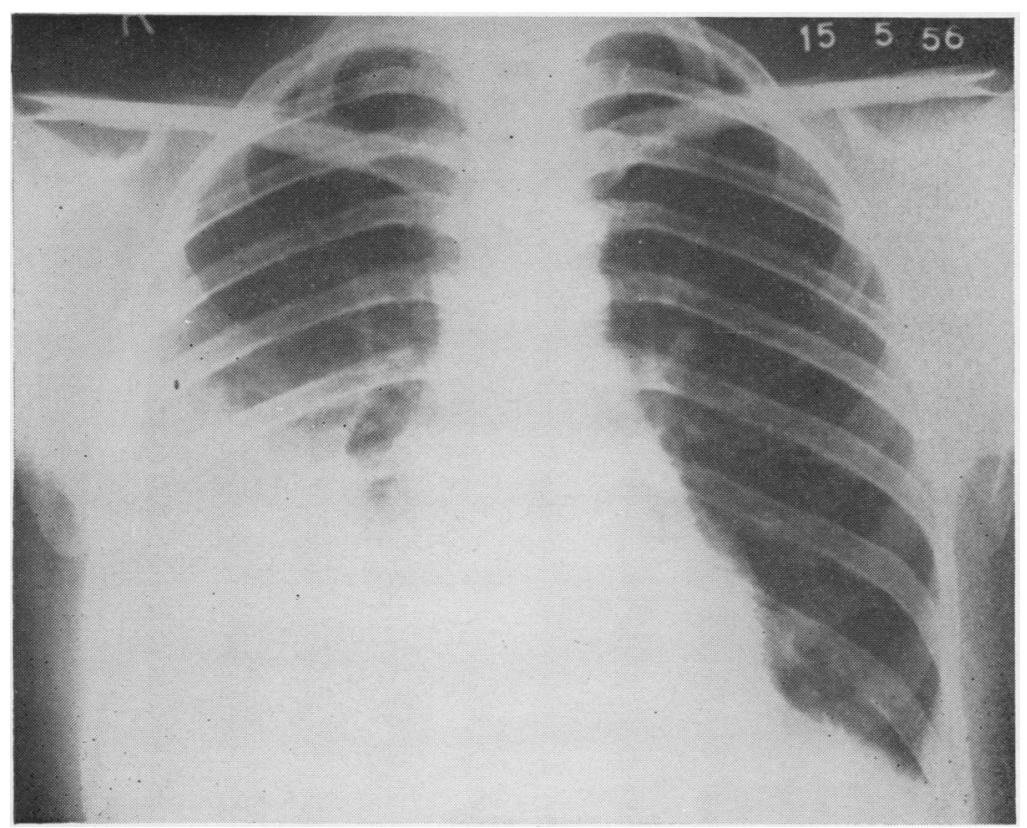

FIG. 1. Case 1. Chest radiograph showing bilateral pleural effusions. 
FIG. 2

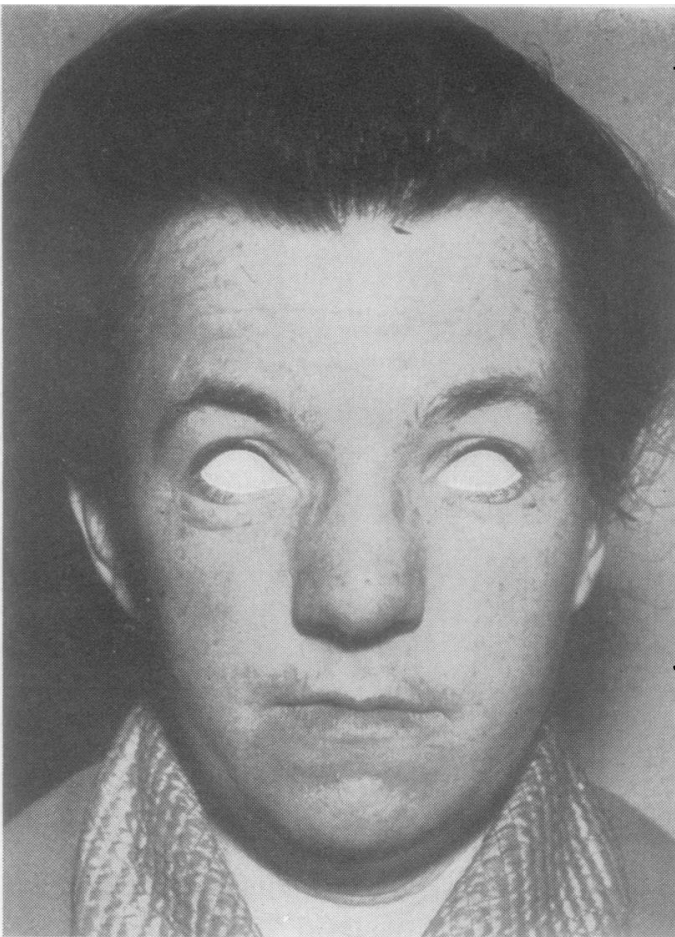

FIG. 4

$\overrightarrow{\overrightarrow{0}}$

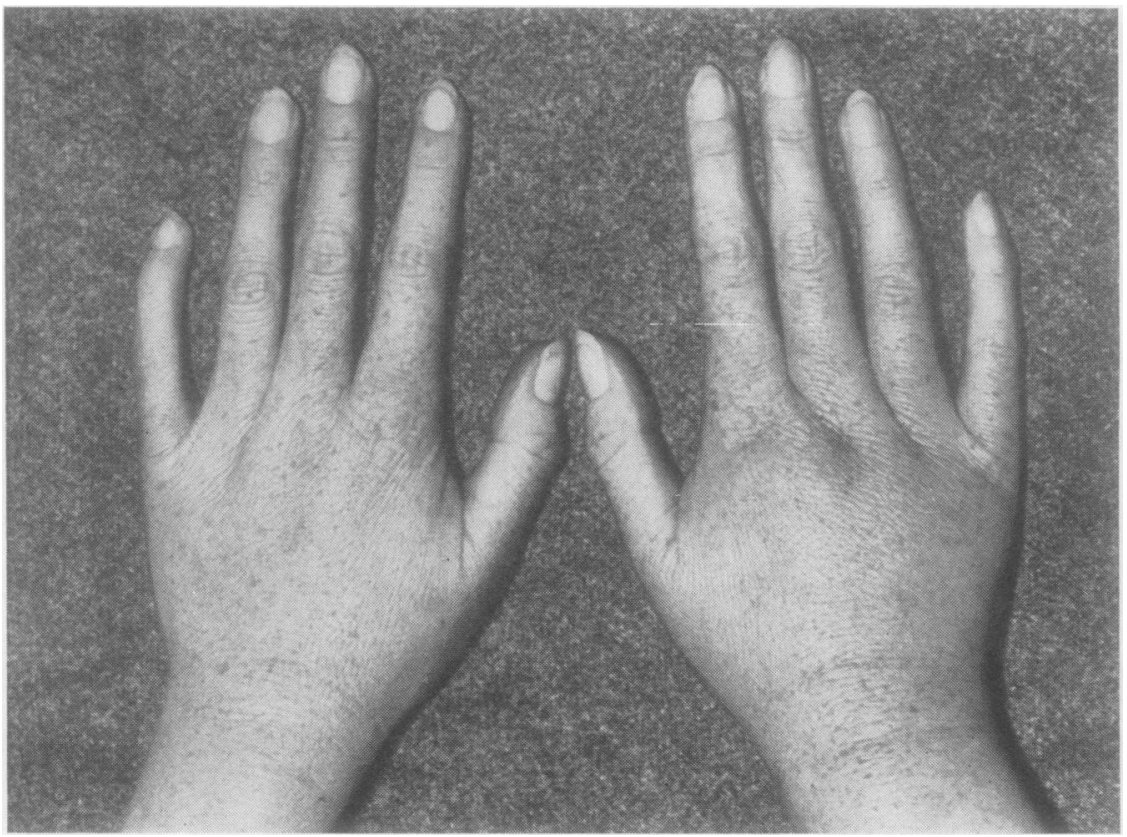

Fig. 3

FIGS 2, 3, and 4. Case 1. Lymphoedema of the legs, hands, and face. S 
over was investigated using radioactive iodinated serum albumin injected into the pleural effusion, but these studies showed only that the exchange of albumin was very slow, as would be expected in any chronic effusion.

In addition to many biochemical investigations, too numerous to detail here, she was studied by bronchoscopy, thoracoscopy, and bronchography, and had several pleural biopsies. None of these revealed any significant abnormality to account for the effusions.

Figures 2, 3, and 4 show the patient at the age of 33 when she was admitted to the Brompton Hospital in 1956. At that time she was not inconvenienced by the swelling of the legs (Fig. 2) and hands (Fig. 3) or the puffiness of the face (Fig. 4), but she was short of breath due to bilateral pleural effusions.

Various diagnoses, including myxoedema, were considered and excluded. In spite of the negative bacteriological and pleural biopsy studies, she was given a therapeutic trial of antimycobacterial therapy on the outside chance that the effusions might be tuberculous in origin. She was treated with streptomycin and isoniazid for three months, but the subsequent behaviour of the effusions was so unlike that seen in cases of tuberculosis of the pleura that this treatment was abandoned. However, in the following year (1957) one out of seven specimens of pleural fluid examined after seven different aspirations was reported positive for acid-fast bacilli on direct smear. Since the organism did not grow on culture, and a guinea-pig inoculation was negative, this finding was discounted.

Subsequently, once in 1958 and again in 1959, positive cultures for tubercle bacilli were returned from among seven separate specimens of pleural fluid examined in those two years. After the second positive culture she was restarted on antimycobacterial drugs in the form of streptomycin and isoniazid, but this made no difference to the reaccumulation of the fluid nor to the clinical condition, and she required repeated aspirations of the effusions. In January 1959 a malignant melanoma appeared on the leg, and, although this was excised, she subsequently developed generalized melanomatosis and died in June 1960 ; there was no necropsy.

CASE 2 Mr. C. E., born in 1913, was well until February 1960, when at the age of 47 he was found to have a right pleural effusion at mass miniature radiography (Fig. 5). As in the first patient the subsequent investigations, which included bronchoscopy, bronchograms, and several pleural biopsies, showed no significant abnormalities to explain the effusion. The pleural fluid was clear yellow material, with a

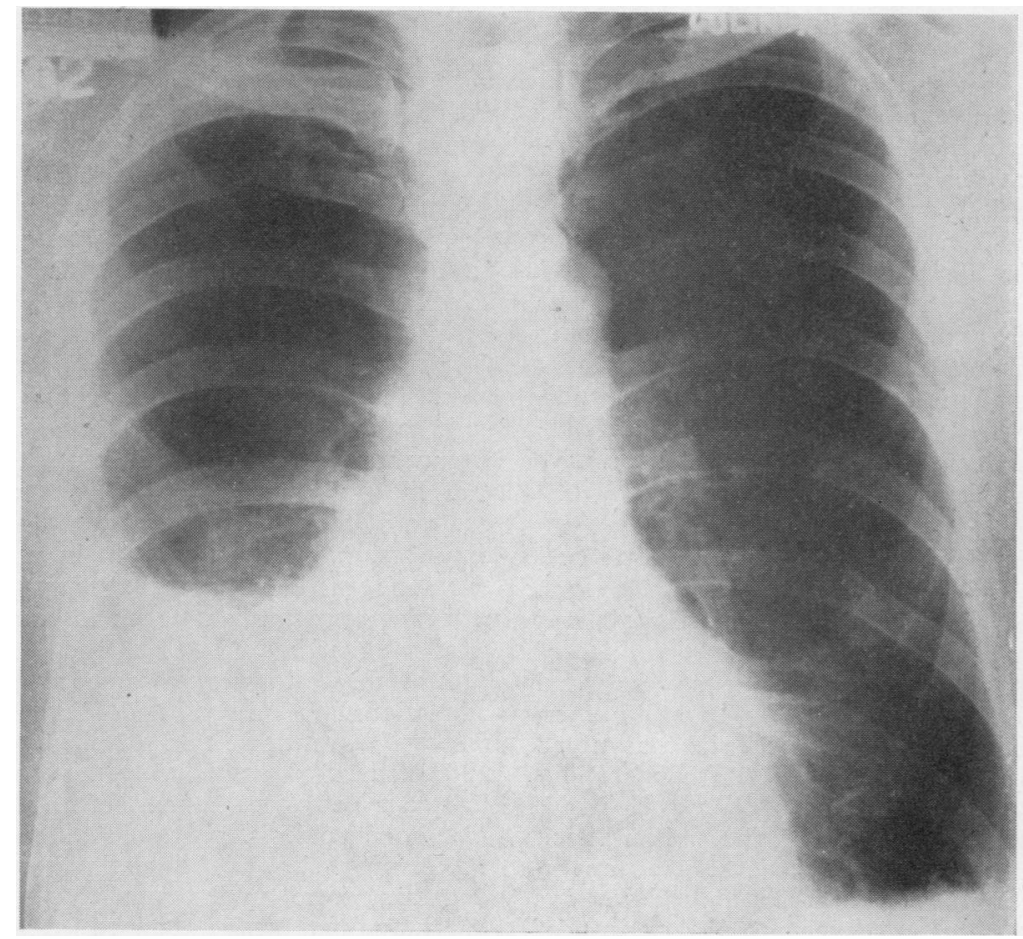

FIG. 5. Case 2. Chest radiographs showing moderate-sized right and very small left pleural effusions. 


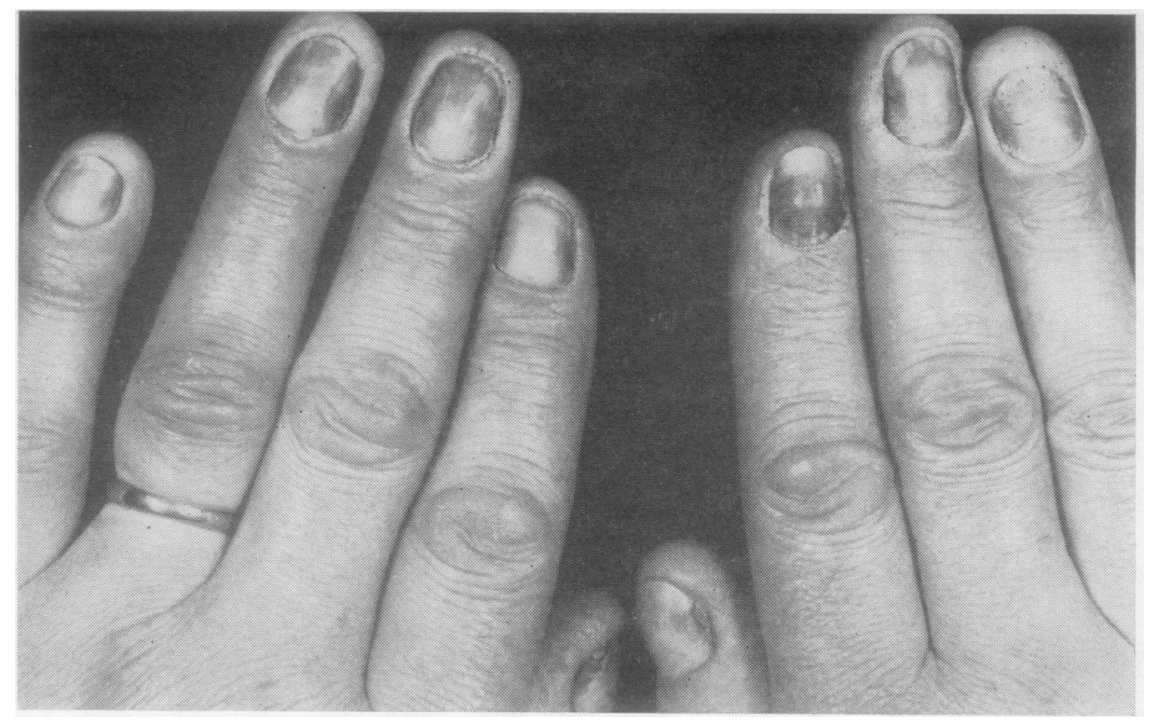

FIG. 6. Case 2. The deformed finger-nails.

protein content of $9 \mathrm{~g} . / 100 \mathrm{ml}$.: the predominant cell was the lymphocyte. The fluid was sterile on culture for both pyogenic organisms and tubercle bacilli. Nevertheless, again on the outside chance that the effusion might be tuberculous in origin, he was treated with para-aminosalicylic acid and isoniazid for two years: this had no effect on the pleural effusion, which remained unchanged in size.

In June 1964, at the age of 51, he was admitted to Westminster Hospital on account of yellow finger(Fig. 6) and toe-nails, when lymphoedema of the legs (Fig. 7) and of the hands was seen. The face showed a certain puffiness (Fig. 8) reminiscent of the first patient (Fig. 4). Lymphangiography showed that the lymphatics in the legs were grossly deficient; there were no megalymphatics. Since that time the condition of the nails, the lymphoedema, and pleural effusion have remained unchanged. There was no family history of lymphoedema.

CASE 3 Miss A. M., born in 1898, first developed lymphoedema of the left leg at the age of 21 years. Her mother, aunt, three cousins, and sister all had Milroy's disease of the late onset type. At the age of 37 the patient's right leg also became swollen, and from this time onwards she usually took diuretics. mostly mercurials. At the age of 54 she was noted to have swelling of the dorsum of both hands, puffiness of the face, and 'very brittle nails'.

In January 1960, at the age of 62 , she had an attack of 'bronchitis with asthma' and developed breathlessness on exertion. In September 1960 there was pain in the left chest with increased shortness of breath: she was admitted to Epping Hospital. In addition to gross lymphoedema (Fig. 9) she was found to have bilateral pleural effusions (Fig. 10). There were no clinical signs of cardiac failure, but the E.C.G. showed evidence of an anterior myocardial infarction with a B.P. of $175 / 95 \mathrm{~mm}$. Hg.

Her recovery from this episode was uneventful, $\overrightarrow{\vec{B}}$ but over the subsequent years she required regular and repeated aspirations of one or two pints of $\frac{3}{2}$ pleural fluid, which was clear, straw-coloured material with a protein content of $4 \mathrm{~g} . / 100 \mathrm{ml}$., and the predominant cell was the lymphocyte. All other biochemical and haematological tests failed to reveal 음 any significant abnormality to account for the $\times$ effusions.

In May 1961, at the age of 63, she was admitted to Guy's Hospital on account of increased breathless- $\bigcirc$ ness. The clinical condition, the bilateral lymphoedema, the pleural effusions, and hypertension were응 all unchanged. On this admission it was again noted that the nails were abnormal and showed 'an overgrowth of the nail-bed and pigmentation of one year's duration'. Nail-clippings were investigated for fungi, $\mathrm{N}$ but none was demonstrated. At this time the bilateral effusions were thought to be in some way related to $N$ the lymphoedema.

Over the following years she had numerous othero admissions to Guy's and Epping Hospitals for repeated aspirations. In 1962 she became unconscious $\frac{C}{\mathscr{D}}$ and died after a period of severe diarrhoea: her plasma electrolytes taken before death suggested a metabolic acidosis.

Necropsy showed bilateral straw-coloured pleural $\stackrel{ }{\mathbb{D}}$ effusions, more on the right than on the left, where $\frac{O}{\square}$ the lung was firmly attached to the chest wall by dense adhesions. The left kidney was small and contracted, the right being twice the normal size. 


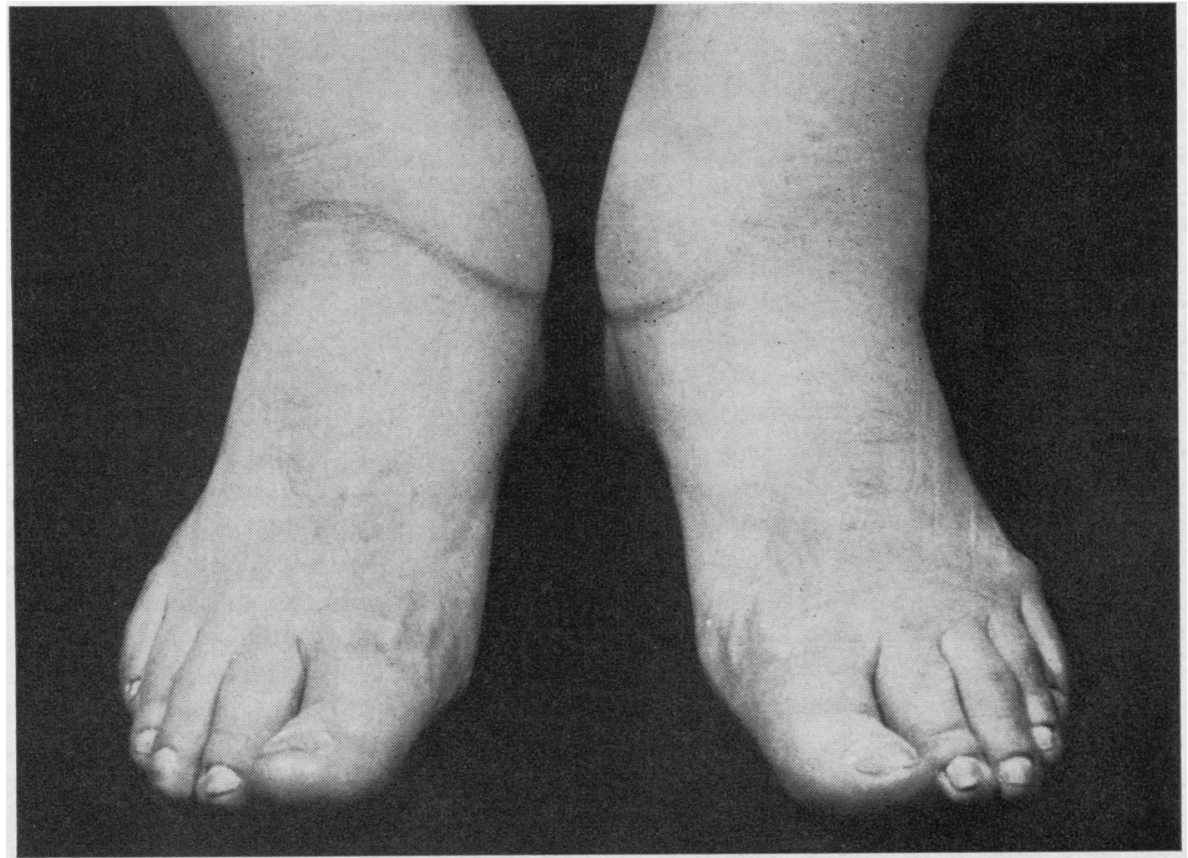

Fig. 7

FIGS 7 and 8. Case 2. Lymphoedema of the legs and face.

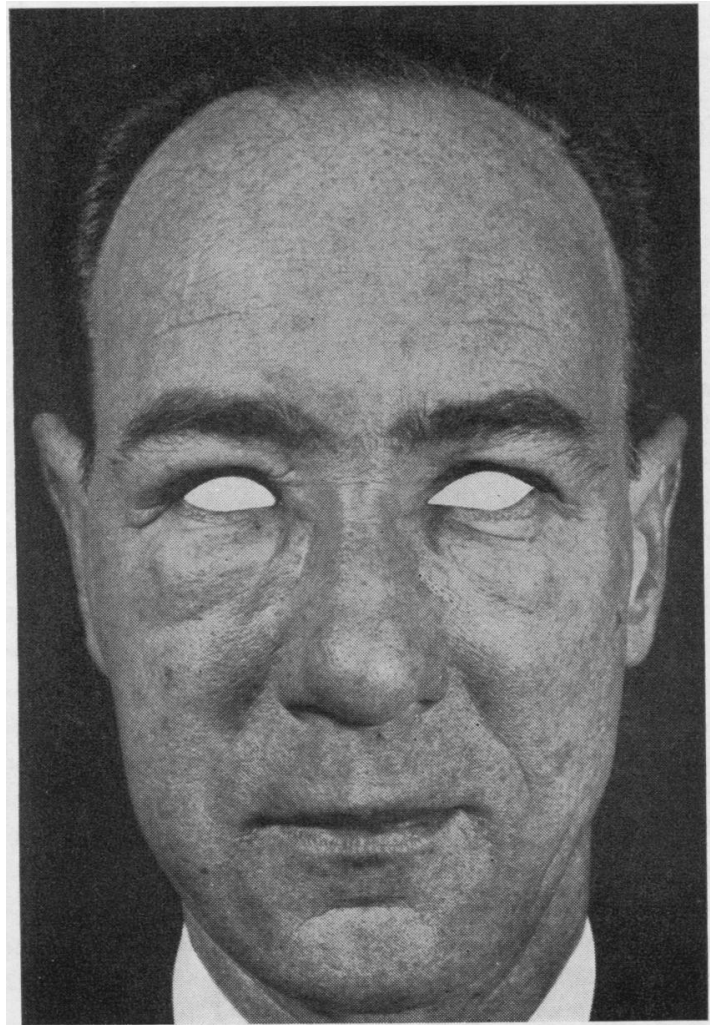

FIG. 8
No other significant abnormalities were found and the brain was normal. It was assumed that death was due to a metabolic acidosis related to the period of diarrhoea. No studies of the lymphatics were made, because at this time lymphoedema as a cause for a pleural effusion had not, so far as I am aware, been described.

\section{DISCUSSION}

The association of lymphoedema with serous effusions in the pleural and peritoneal spaces was described by Morris, Blood, Sidman, Steel, and Whittem (1954) in newborn Australian Ayrshire calves, and the three present patients probably represent a related condition. All three were suffering from lymphoedema not only of the legs but also of the hands and face. The puffy appearance of the face (Figs 4 and 8) was surprisingly similar, and in both the female patients it led to the serious consideration of a diagnosis of myxoedema. Case 3 was in fact treated for a period with thyroid extract without improvement: objective thyroid function tests in both the patients were normal. The effusions, at least in cases 1 and 3, apparently appeared after a lower respiratory infection described as 'bronchitis' in case 1 and as 'bronchitis and asthma' in case 3.

It is well recognized that patients with a congenital deficiency of the lymphatics often do not develop persistent lymphoedema until after some infection or other episode such as an insect bite 


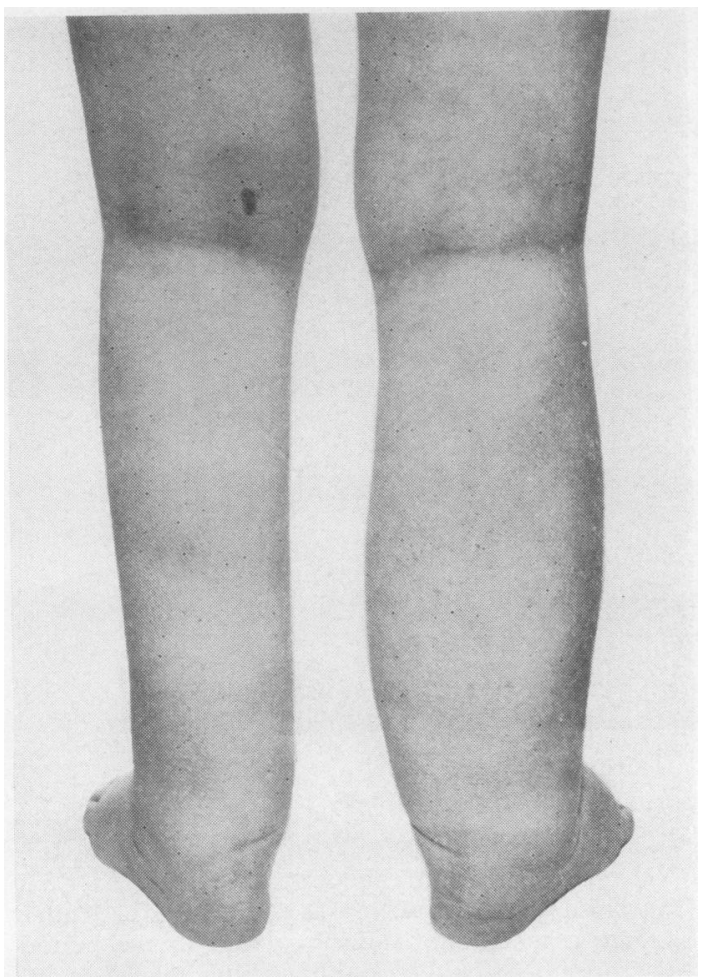

FIG. 9. Case 3. Lymphoedema of the legs. or injury. This increases the local capillary perm $\vec{F}-$ ability and throws an increased load on the already deficient lymphatics, which are unable to drati off sufficient protein, and so oedema occurs a ajod persists. The same mechanism may operate in $\widetilde{\Phi}^{a}$ pleural space, where a lower respiratory or pleural infection may damage a previously adequäee lymphatic drainage, and so result in a persiste $\vec{b} t$ pleural effusion. Although the effusions in case-1 came after a lower respiratory infection it is dif cult to discount the two later positive cultures for tubercle bacilli. Certainly this patient's clinie course, the thoracoscopic appearances, and the way in which the effusions persisted for yexps without the development of pleural thickening was quite unlike the usual behaviour of tuberculouls effusions. Perhaps a small tuberculous focus $\frac{9}{\mathrm{Pn}}$ the pleura initiated the original effusion, but the subsequent remarkable persistence of the effusioकोs must have been conditioned by the impaired lymphatic drainage associated with a generalized lymphatic deficiency.

The abnormal, thickened, pigmented yell\& nails in cases 2 and 3 are of special interest. Begh patients were at one time considered to be sufforing from a fungus disease of the nails, but aूn neither case were fungi found in the nail-clippin \&s, and they were both undoubtedly examples of 'yellow-nail syndrome' of Samman and White

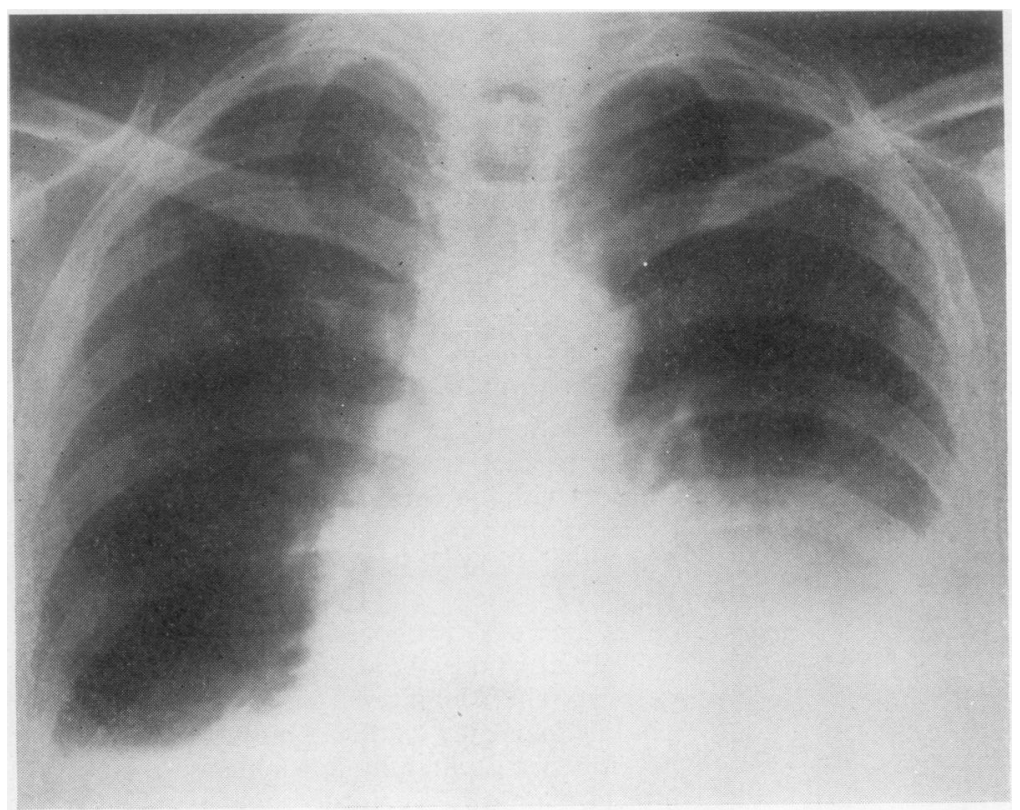

FIG. 10. Case 3. Chest radepsgraphs showing bilateral pleural effusions. 
(1964). These authors first described the association of slow-growing discoloured yellow nails with lymphoedema. The nails are usually smooth, but may show transverse ridging and are usually excessively curved from side to side, so that coverage of the lateral ridges by the surrounding soft tissue is less than normal and the cuticles are deficient. The colour is usually pale yellow, but may be slightly greenish. Nail separation may occur and some partially separated nails have a distinct hump. Although the nail changes are believed to be the result of defective lymphatic drainage, evidence of lymphoedema may not appear until years after the finger-nails or toenails have become abnormal.

Minor and even moderate degrees of lymphoedema are easily overlooked. The patient may have had the condition for so long that he has come to regard it as almost normal, so that no specific complaint is made, and the evidence may remain hidden under the bed-clothes when the chest or nails are examined. It is important, therefore, in chronic unexplained pleural effusion to look for evidence of impaired lymphatic drainage ; this may be obvious in the legs, hands, and even face, but it is also important to look for the slowly growing deformed yellow nails which may indicate impaired lymphatic drainage even in the absence of obvious lymphoedema. Although such cases have not been encountered, if a patient with a chronic unexplained pleural effusion were found to have deformed yellow nails without obvious evidence of lymphoedema it would be wise to carry out lymphangiography of the legs to see whether there was any evidence of lymphatic deficiency or megalymphatics.

The three present patients all had a great deal of investigation and treatment which proved to be irrelevant when the relationship of the pleural effusion to the lymphoedema was eventually realized. None of them had any specific treatment to prevent the recurrence of pleural effusions. Case 2 had only a small effusion which did not require aspiration, but cases 1 and 3 were both severely handicapped and distressed by bilateral effusions, which required the frequent removal of large quantities of pleural fluid.

In case 3 necropsy showed that the left pleural space was largely obliterated by adhesions, presumably explaining why the effusion was small and not troublesome on that side. The rational treatment of future cases would appear to be pleurodesis by thoracotomy and parietal pleurectomy.

\section{SUMMARY}

Three patients with chronic and otherwise unexplained pleural effusions are described and the condition is attributed to a deficiency of the lymphatics draining the pleural space. All three had chronic lymphoedema of the legs, hands, and face: two of them had abnormal yellow nails which are also believed to be due to deficient lymphatic drainage.

I am most grateful to Dr. K. Robson and Dr. C. G. Baker for permission to report cases 1 and 3 ; to Dr. R. Grenville Mathers and Dr. P. Samman for permission to report case 2 , and particularly to Dr. P. Samman and the Institute of Dermatology for allowing me to reproduce photographs of case 2 and of his nails.

\section{REFERENCES}

Hurwitz, P. A., and Pinnals, D. J. (1964). Pleural effusion in chronic hereditary lymphedema. (Nonne, Milroy, Meige's disease.) Radiology, 82, 246.

Morris, B., Blood, D. C., Sidman, W. R., Steel, J. D., and Whittem, J. H. (1954). Congenital lymphatic oedema in Ayrshire calves. Aust. J. exp. Biol. med. Sci., 32, 265.

Robson, K., and Emerson, P. A. (1963). In Chest Diseases, ed. K. M. A. Perry and T. Holmes Sellors, Vol. I, p. 194. Butterworths, London.

Samman, P. D., and White, W. F. (1964). The 'Yellow Nail' syndrome. Brit.J. Derm., 76, 153. 